\title{
THE TYPES OF COMPUTER GRAPHICS AND THEIR APPLICATION AT DIFFERENT LEVELS OF KNOWLEDGE
}

\author{
Anna Makarewicz' ${ }^{1}$ Sylwester Korga ${ }^{2}$, Wojciech Rosa ${ }^{1}$ \\ 1 Department of Applied Mathematics, Lublin University of Technology, Nadbystrzycka 38, 20-618 Lublin, \\ Poland, e-mail: a.makarewicz@pollub.pl; w.rosa@pollub.pl \\ 2 Department of Fundamentals of Technology, Lublin University of Technlogy, Nadbystrzycka Str. 38, 20-618 \\ Lublin, Poland, e-mail: s.korga@pollub.pl
}

Received: 2015.10.11

Accepted: 2015.11.14

Published: 2015.12.04

\begin{abstract}
In this article we introduce the concept of computer graphics and graphical application C.a.R (Compasses and Ruler), its basic commands and several examples associated with the geometry. This subject is at all levels of knowledge in various stages of development. We will present the C.a.R possibilities that can be used in secondary schools, high schools and colleges. We show function graphs of varying degrees of difficulty that are too complicated for the human imagination.
\end{abstract}

Keywords: visualization, dynamic geometry, teaching.

\section{INTRODUCTION}

The world development and rapid growth of the Internet in combination with its increasing accessibility for the public have opened up a whole new digital world. During learning process students are increasingly inclined to accept the content given to them in this way. Especially in mathematics where a number of issues require a lot of imagination, students are discouraged to learn the subject when it is not provided in a modern and accessible way [4]. We show how to use computer graphics to teach mathematics. A computer is an excellent educational tool used to teach geometry. The first attempts to solve geometric tasks on the computer surprise both students and teachers. It occurs that even complete lack of programming skills is not an impediment for the teachers to confirm the theory presented during the lecture by a computer presentation [3].

Computer graphics - a part of computer science that uses computer techniques for artistic visualization and visualization of reality. Computer graphics is a tool commonly used in science, technology and entertainment. We accept the year 1951 as a birth date of computer graphics. In this year the first device was built, which was used to display the results of computing in a visual form (Massachusetts Institute of Technology).

\section{METHODS FOR DISPLAY IMAGES}

\section{Bitmap graphic}

The images are composed of points of the same size (pixels) arranged evenly in rows and columns (grid raster). Each pixel (actually the information about the color) can be saved by using a specified number of bits; a value of 1 means black or color, value 0 white. We distinguish maps: 1-bit, 8-bits, 16-bits, 24-bits and 32-bits, it depends on the number of colors that can be used in the bitmap. 1-bit maps are black and white maps. In the 8-bit maps to one pixel falls 256 colors, 16bit 65526 colors, 24-bit 16777216 colors.

Advantages: rich colors, freely contrast matching, brightness, color saturation and the possibility to use complex tools for processing digital photographs, the possibility of retouching, correction, various forms of montage (photomontage), the possibility of applying multiple effects, deformation, inserting texts, etc. 
Disadvantages: the impossibility of scaling without losing quality, at high magnification the image quality is miserable.

Application: digital image processing, color documents, brochures and technical drawings formation etc.

\section{Vector graphics}

Creating pictures of elements (vectors) described by the mathematical equations which describe their position, size, orientation, etc. Vectorial image is a set of vectors. All graphical elements (objects) are stored separately, so you can easily edit, modify, copy, etc. There is no concept of resolutions related to the number of pixels (picture quality depends on the resolution of the printer).

Advantages: Scaling does not decrease image quality. It is also easy to edit the image. A small size of files, the ability of changing images parameters, a good conversion to raster graphics, a good description of three-dimensional spaces.

Disadvantages: impossible detailed editing of images (with respect to the pixel).

Uses: text graphics creating (business cards, logos), coats of arms, flags, emblems, scientific and technical diagrams, maps and plans, various types of signs, eg. road, some graphic artistic creation (eg. the comics).

Geometry and its transformation constitute a description of three-dimensional space which is described by methods of vector graphics. Appearance of the objects is determined by raster graphics called textures.

There are various articles of geometric solutions tasks which are performed in the classroom with using computer programs like Geogebra [4], Cabri [5], the Wolfram Demonstrations Project platform [3]. The program C.a.R (Compasses and Ruler) is used to create geometric constructions on the plane using the "simulators" of a compasses and a ruler. C.a.R is free, and its possibilities combined with ease of use make it a good tool for exploring geometry.

\section{C.A.R PROGRAM POSSIBILITIES}

The C.a.R application toolbar allows us to draw almost every kind of shape. It was programmed by profesor of mathematics Rene Grothmann and is used by him to teach students geometry at Die Katholische Universitat Eich-
statt-Ingolstadt at the Faculty of Mathematics and Geography. C.a.R. is an advanced program for learning geometry. The main purpose of the program is to teach geometry on a plane. The program requires only Java and therefore it runs on any operating system that supports this technology.

The simplest objects are obviously the points that we set by clicking in the space of the plane. Straight line, half-lines and segments we draw by pointing to two points (if there is no point in the space it will be created). We can draw a circle in two ways: indicating the center and point on the circle or center and two points defining the radius. All points created after drawing can be moved. It is easy to construct a perpendicular line and parallel line.

Another feature of the program is the ability to find and select the intersection point of a large number of functions or objects. Changes in the position of one of the objects cause e.g. the change of intersection point position. In this way we are able to dependence the shape, position and even the existence of a figure from another (Fig. 1).

For example, if we draw a triangle and construct a shell on it, then by changing a single point of the triangle we will change the whole structure of the shell. The teacher can graphically illustrate the influence on the result of construction when individual elements are changed. This can be used to graphic proofs of mathematical theorems. Changing colors, thickness of the pen, signing figures and hiding unnecessary elements, we can create complex and very clear drawings.

C.a.R makes possibility to operate with macroinstruction, which greatly facilitates the creation of complicated structures through their memorization and automatic playback of the structures necessary for drawing the desired figure / object. Macroinstructions parameters are objects created by using the available application tools. For example, we construct a triangle, and other figures which help teachers prove the Pythagorean theorem. This drawing makes teachers job much easier because it will be enough to discuss this drawing instead of drawing it in the board in the classroom and discuss at the same time (Fig. 2).

This program can be used not only in primary schools but also in secondary schools and high schools. You can use it to show some difficult graphs of functions given in a parametric form. Rosette is given by $x=2 a \sin (t) \cos ^{2}(t), y=2 a \sin ^{2}(t)$ $\cos (t)$, let $a=1$, then the graph is as follows (Fig. 3). 


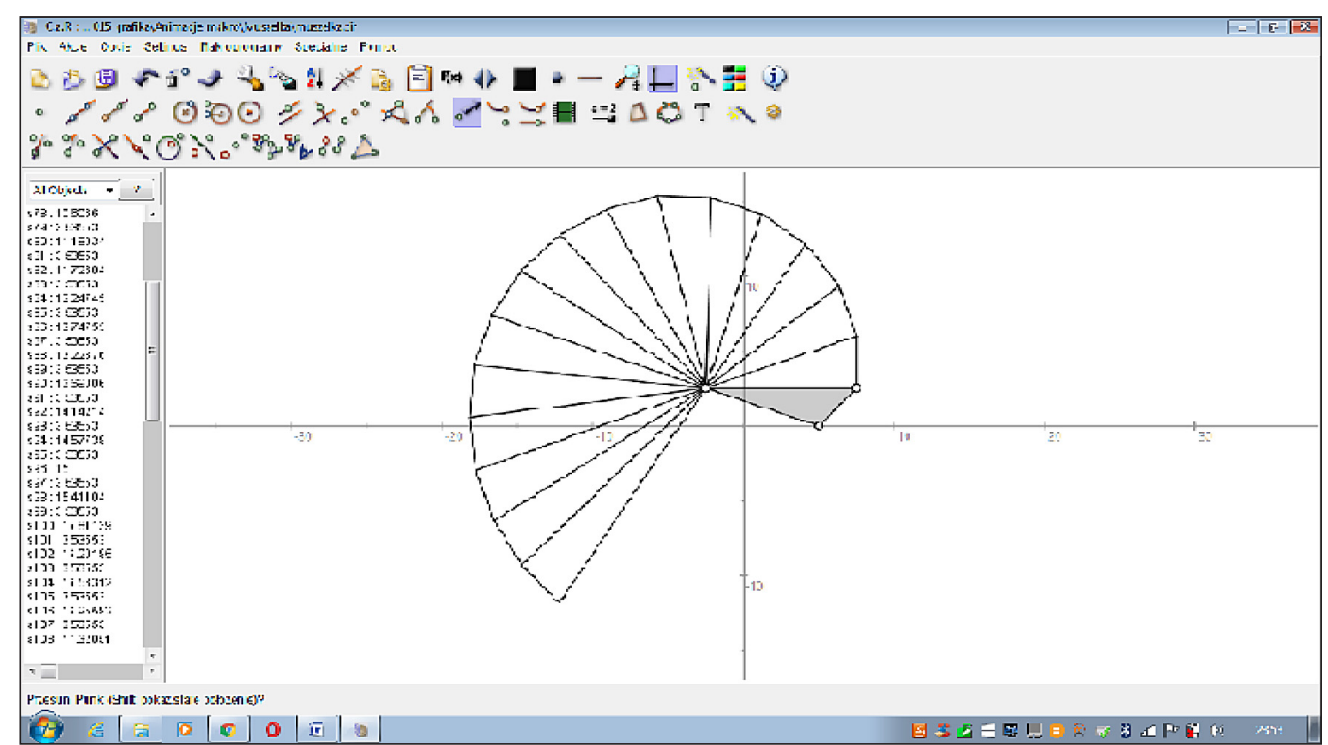

Fig. 1. Macro demonstration - shell

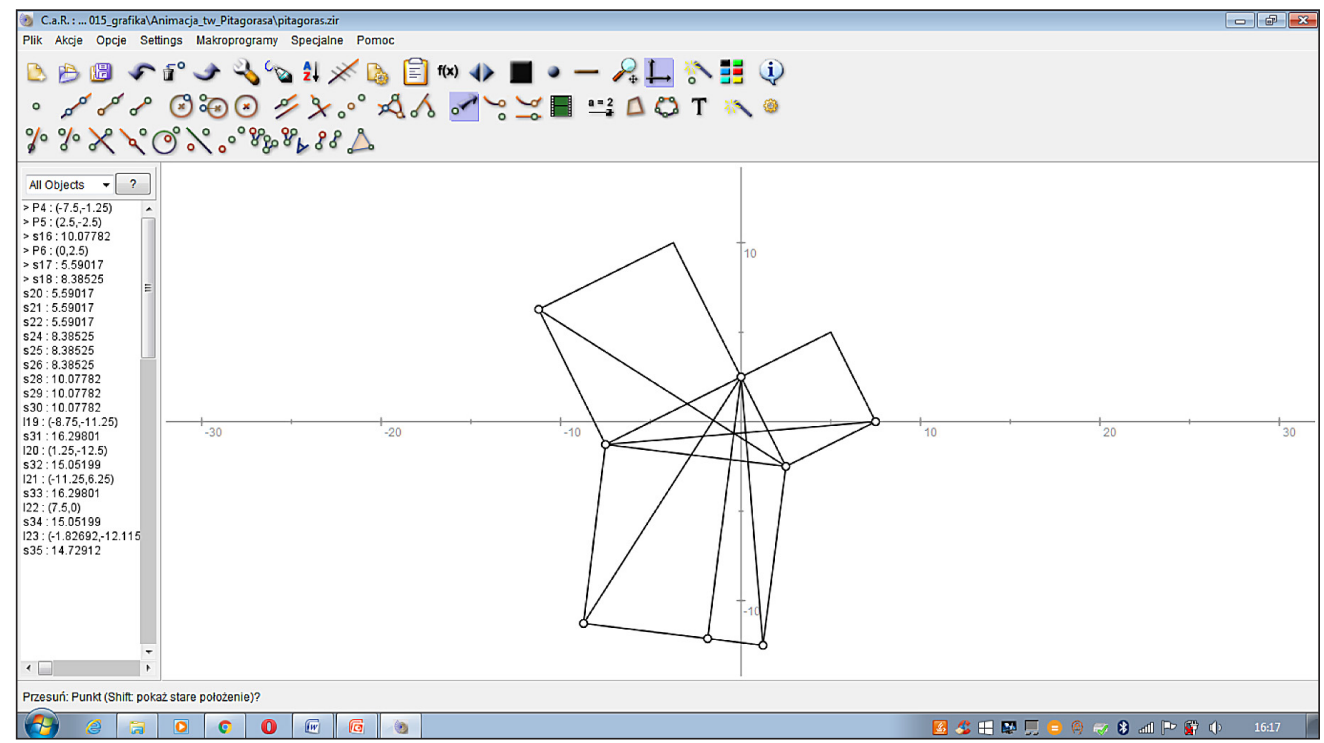

Fig. 2. Macro demonstration - Pythagorean theorem

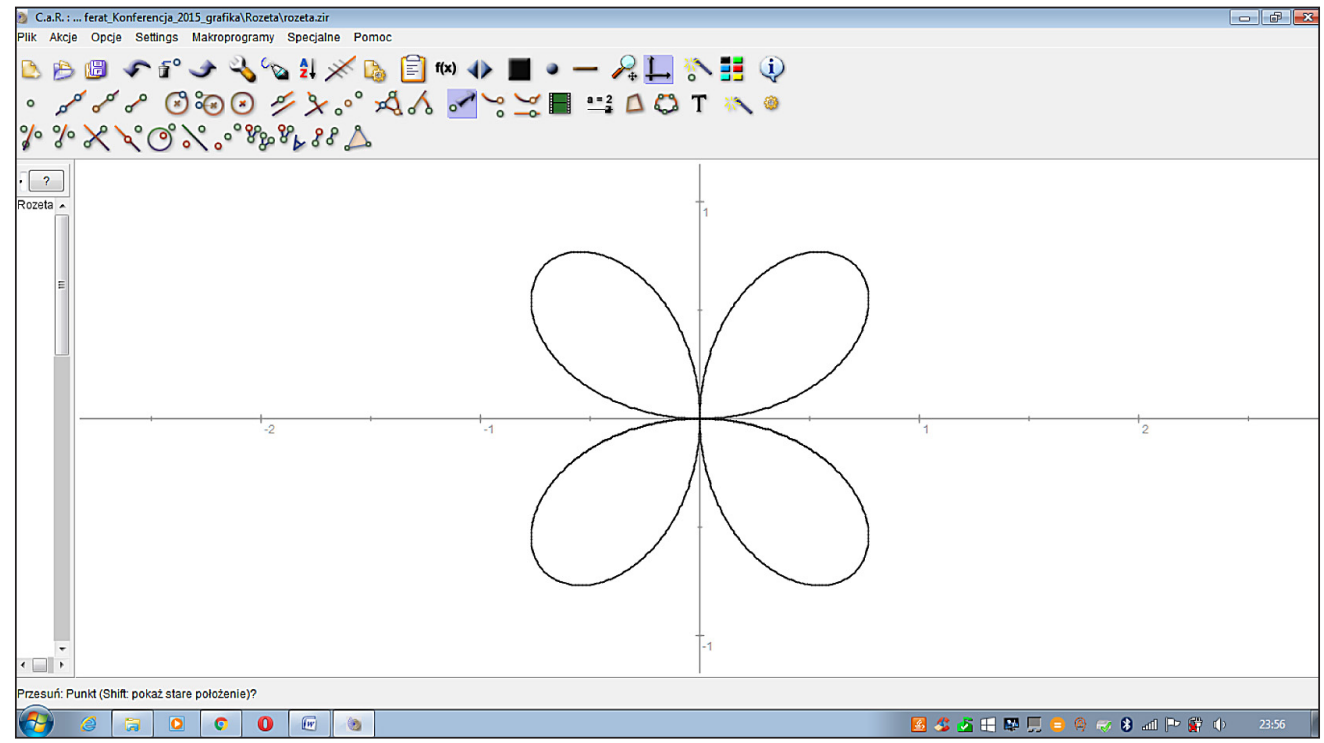

Fig. 3. Rosette 


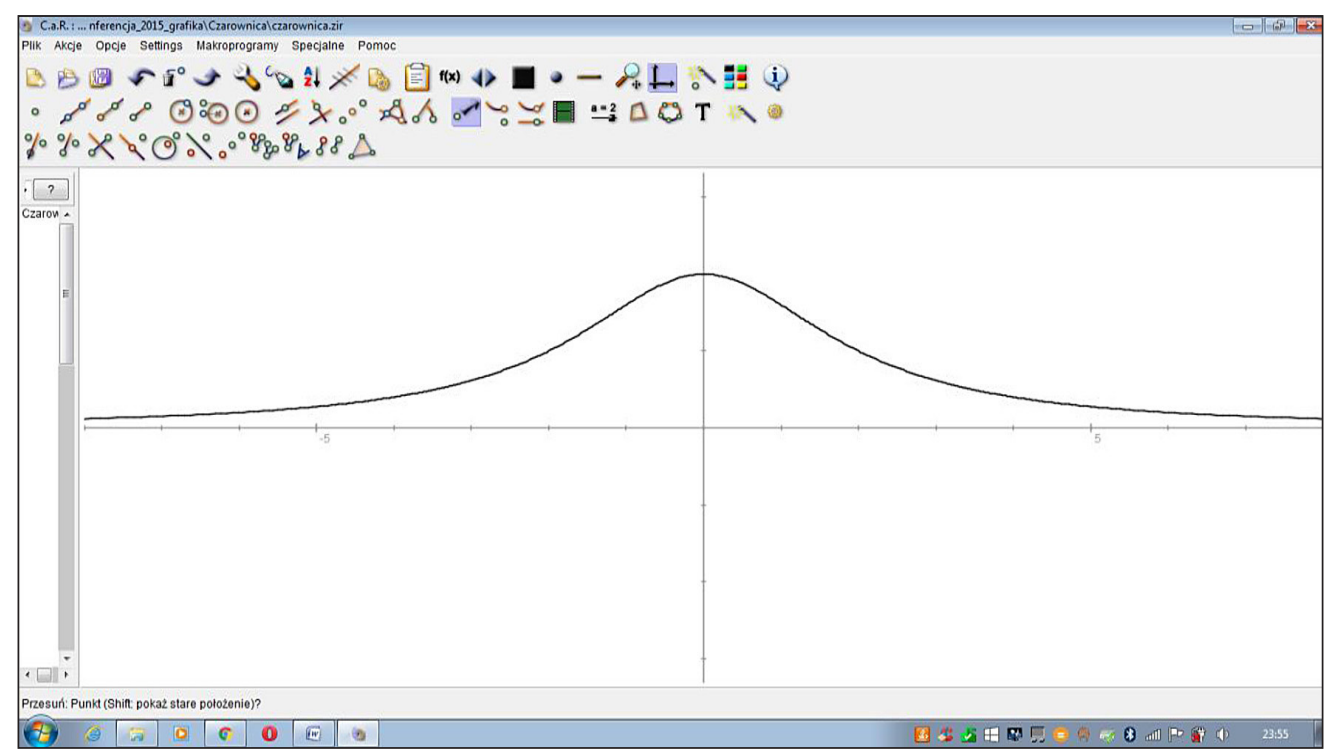

Fig. 4. Graph of function called "witch"

Another difficult graph to draw is a graph of the function commonly called a "witch" defined by formula $f(t)=\left(2 a \operatorname{tg}(t), 2 a \cos ^{2}(t)\right)$ let $a=1$. Unfortunately when we see such formula, our imagination isn't able to help us to draw this kind of graph, definitely it would take us a lot of time (Fig. 4).

It is worth mentioning that the program makes it possible to trace the whole structure step by step by displaying a sequence figures we made. This gives us the possibility to use the program on computer science lessons as an illustration of simple algorithms.

\section{CONCLUSIONS}

Creating dynamic construction or Winding geometric lotus are the two most interesting features of programs supporting teaching of geometry. Graphics generated by computer techniques are applicable in many fields related to the design of industrial products. We generate a visualization whose quality resembles images of photographic, in this way the potential buyer is able to see the finished product before it is made. In this way a huge amount of graphics are created to represent different elements of our daily life, from keychain to skyscrapers.
Lessons which are conducted by using the program C.a.R really stimulate the imagination of students and their interest in both the geometry and the use of computers for learning mathematics. Using that mathematical software increases students' interest in mathematics, information technology and improves their skills [5]. The program CaR offers great possibilities, and its interface is simple enough that students with proficiency in working on Windows need just one hour lesson to learn it.

\section{REFERENCES}

1. https://pl.wikipedia.org/wiki/Grafika_komputerowa. [Accessed 28.10.2015].

2. Gajda W., Description of C.a.R program [Online]. http://www.gajdaw.pl/geometria/c-a-r-opis/print. html\#R7. [Accessed 28.10.2015].

3. Gorgol I., Wolfram demonstrations project platform as a support in teaching. Adv. Sci. Technol. Res. J., 9(26), 2015, 143-147.

4. Majerek D., Application of Geogebra for teaching mathematics. Adv. Sci. Technol. Res. J., 8(24), 2014, 51-54.

5. Rososzczyk R., Application of Cabri 3D in teaching stereometry. Adv. Sci. Technol. Res. J., 9(26), 2015, 148-151. 